\title{
TIIEODOR WEYL.
}

Um die Mitte des vorigen Jahrhunderts lebte in Berlin ein Dr. phil. Louis Weyl, dessen Name in den literarisch interessierten Kreisen jener Zeit wohlbekannt war. Er schrieb neben praktischen Handbüchern ${ }^{1}$ ) besonders auch scherzhafte Bücher und Aufsätze im Stile seines der Nachwelt länger bekannt gebliebenen Zeitgenossen Glasbrenner, die er mit außergewöhnlichem Heiterkeitserfolge den lachfrohen Berlinern mündlich-vorzutragen pflegte. Als er im Jahre 1852 32-jährig und kaum $\mathrm{z}$ wei Jahre nach seiner Verheiratung (mit Lina geb. Weinberg) starb, hinterblieb seine junge Wittwe mit einem am 8. Januar 1851 geborenen Sohne Theodor, von dem in diesen Zeilen weiter die Rede sein soll. Ein zweiter Sohn Louis wurde bald nach des Vaters Tode geboren. Mit diesem zusammen wuchs Theodor, der ein schwächliches Kind war, zunächst nur unter mütterlicher Obhut, dann unter Leitung eines Hauslehrers auf und kam erst spät in eine öffentliche Schule. In den Jugendjahren, wo die Schulwisssenschaften nur einen Teil seiner Zeit in Anspruch nehmen sollten, wurde die Phantasie und Wißbegier des lebhaften Knaben besonders durch Pflanzenkunde und Musik gefesselt, denen er schon damals eine Liebe widmete, die ibn durch das Leben begleitet hat. In der späteren Gymnasialzeit trat dazu ein eingehendes Interesse für die klassischen Schriftsteller des Altertums, das ihn bis an sein Lebensende befähigte, zahlreiche Stellen aus ihren Schriften in der Unprache frei aus dem Gedächtnis zu zitieren. Trotzdem oder vielleicht gerade wegen der einseitigen Bevorzugung seiner Lieblingsfächer war Theodor kein sogenannter guter Schüler und bestand erst mit zwanzig Jahren sein Abiturienten-Examen.

1) »Fremdenführer für Berlin und Potsdam", *Wegweiser für EisenbahnReisende durch Berlin, Potsdam, Dessau, Köthen, Halle, Magdeburg, Leipzig, Dresden oder: Wie kann der Reisende in acht Tagen alles Sehenswerte dieser Städte, ihre Umgebungen und die sie verbindenden Eisenbahnen kennen lernen? $\alpha$ usw.

Borichto d. D. Chem. Gegellschaft. Jahrg. XXXXVII. 


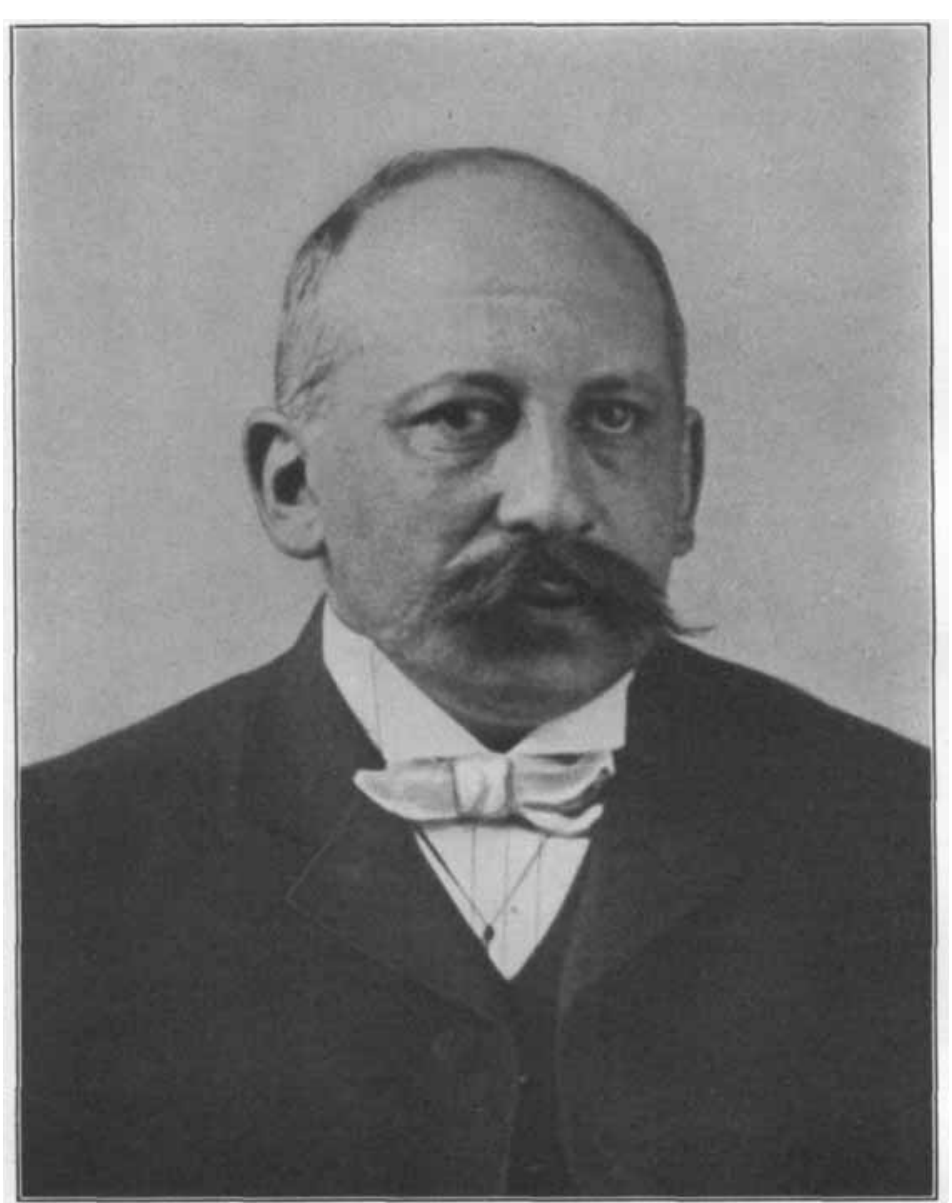

thulene 
Die Universität bezog er zuerst in Hejdelberg, dann in Berlin und schließlich in Straßburg und widmete sich mit Erast und Eifer dem Studium der Medizin und Cbemie, führte daneben aber im Kreise gleicbstrebender Altersgenossen ein sebr lustiges, oft ausgelassenes Studentenleben. Einer der Freunde aus jenen Jahren schildert ibn mit den Worten: 'Schon damals waren an ihm zwei Eigenschaften ausgeprägt: ein burschikoser Humor, der oft an Übermut grenzte, und ein ganz ungewöhnliches Interesse an allen naturwissenschaftlichen Fragen, in denen er sich. sehr bewandert zeigte. Er steckte voll studentischen Übermuts, liebte Neckerei und Spott, war aber doch gutmütig und gegen seine näheren Bekannten berzlich und treu. Am Studium interessierte ibn nach wie vor das streng naturwissenschaftliche, insbesondere Physiologie und Chemie, so da $B$ er namentlich an Hoppe-Seyler sich anschloß. Mit den klinischen Fächern hat er sich nie recht befreundet - er hatte für das ärztliche, sowohl was Krankenbeobachtung, als was Therapie anbetraf, keinen rechten Sinn, weil ihn hierbei der Mangel wissenschaftlicher Exaktheit störtec.

Noch als Student veröffentlichte Weyl aus den Instituten $d u$ BoisReymonds in Berlin und Hoppe-Seylers in Straßburg einige kleinere Untersuchungen physiologischen Inhalts, bestand an letzterer Universität 1877 sein Doktorexamen - die Dissertation betral *Beiträge zur Kenntnis tierischer und pllanzlicher Eiweißstoffe - und im folgenden Jahre ebenda sein medizinisches Staatsexamen.

Dann nach Berlin zurückgekehrt, trat er in das unter E. Baumanns Leitung stehende chemisch-physiologische Universitäts-Laboratorium ein und veroffentlichte als Resultat seiner dortigen Studien unter dem Titel sÜber eine neue Reaktion auf Kreatinin und Kreatin ${ }^{1}$ ) die unter seinem Namem sebr bekannt gewordene Farbenreaktion auf Kreatinin mit Nitroprussidnatrium in alkalischer Lösung, sowie eine Beobachtung > Ưber Spaltung von Tyrosin durch Fäulnis* ${ }^{2}$ ), worin er nachwies, daß der vorher von Bauman n bei der Einwirkung der -Bauchspeicheldrüse auf Eiweiß erbaltene Phenolkörper $p$-Kresol ist, welches sekundär aus vorher abgespaltenem Tyrosin entatanden zu denken ist; er gewann das Kresol aus dem Tyrosin im Brütofen unter Einwirkung von $>$ Pankeschlamma aus dem damals noch durch seine Unreinheit berüchtigten Nebenflüßchen der Spree.

Diesen frühen Eriolgen verdankte er es wohl, daß er noch im glẹichen Jahre die Assistentenstelle an dem unter I. Rosenthals Leitung stehenden physiologischen Universitäts-Institut in Erlangen

1) B. 11, 2175 [1878].

2) H. 3, 312 [1878]. 
erhielt und zugleich von der dortigen medizinischen Fakultät als Privatdozent zugelassen wurde. Seine Habilitationsschrift »Über Eiweißverdauung und über Spaltung des Tyrosins durch Fäulnis« beruht auf der oben erwähnten Beobachtung. Kurze Zeit darauf verheiratete er sich mit seiner Cousine Elise, geb. Weinberg, der Tochter eines Danziger Großkaufmanns, in dessen Hause der junge Gymnasiast und Student fast alle seine Ferien verlebt hatte.

Nun folgten einige Jahre reger wissenschaftlicher Arbeit. Weyl veröffentlichte aus Erlangen, zum Teil in Gemeinschaft mit Schülern, Untersuchungen über: "Die Ausscheidnng von Hippursäure und Ben-

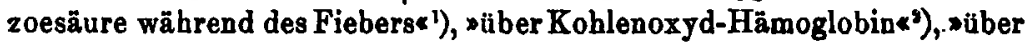
den Kleber* ${ }^{3}$ ), wüber die Sauerstoffabsorption des Pyrogallols in alkalischer Lösung « ${ }^{4}$ ), uüber den Sauerstoffgehalt des Wassers, verglichen mit der Menge der darin enthaltenen organischen Substanzen* "), über den Ammonialgehalt der Grundlufte $\%$, süber die Darstellung ron metallischem Kupfer für die Elementaranalyse* ${ }^{7}$ ), für welchen $Z$ weck er die Ameisensäure als Reduktionsmittel vorschlug, aäber den Einfluß chemischer Agenzien auf die Assimilationsgröße grüner Pflanzen ${ }^{8}$ ), »über die saure Reaktion des tätigen Muskels und über die Rolle der Phosphorsäure beim Muskeltetanus “ ${ }^{9}$ ).

In diese Zeit - Winter 1880-1881 - fällt auch ein längerer Aufenthalt in Neapel, wo Weyl durch seine Verbindung mit du Bois-Reymond und im Auftrage der Berliner Akademie der Wissenschaften einen Arbeitsplatz in der damals noch jungen Zoologischen Station Dohrns erhalten hatte, um über das elektrische Organ des Zitterrochens (Torpedo) zu arbeiten. Die Früchte dieser sphysiologischen und chemischen Studien am Torpedo* legte er in einer Reibe ron Abbandlungen ${ }^{10}$ ) nieder, daneben aber bildete dieser Neapeler Aufenthalt des jungen Forschers eine der schönsten Erinnerungen

1) H. 4, 169 [1879].

7) Mit B. v. Anrep, B. 13, 1294 [1880].

3) Mit Bisch off, B. 18, 367 [1880].

5) Mit X. Zeitler, A. 205, 255; B. 14, 2659 [1881].

3) Mit X. Zeitler, H. 5, 10 [1880].

6) Mit L. Rinck, Inaug.-Diss. Erlangen 1880.7 7) B. 15, 1139 [1882].

g) Sitzgsber. d. Physik.-medizin. Gesellsch. zu Erlangen, Sitzung rom 1. 8.1881 .

9) Mit X. Zeitler, H. 6, 557 [1882].

10) Archiv f. Anatomie u. Physiologie 1888, 117, Suppl.-Band pg. 105, 1884, 316; H. 7, 541 [1883], 8, 316 [1884], 11, 525 [1887]; Ber. d. Kgl. Akademie d. Wissensch, Berlin, rom 21. 4. 1881; Arch. f. Physiol. 1884, 416; Zentralbl. f. d. medizin. Wissenseb. 1882, Nr. 16. 
seines Lebens; der anregende Verkehr in dem Gelehrtenkreise, dessen Mittelpunkt Dohrn war, die groBartige Natur der herrlichen Golfstadt mit ihren historischen Erinnerungen, das interessante Volksleben machten aut Weyls für alle diese Dinge in hohem Maße empfängliches Gemüt bleibend tiefen Eindruck und trieben ihn in späteren Jahren, so oft er es irgend ermöglichen konnte, zu neuen Besuchen nach der Stadt des Vesuvs, die dann auch nicht nur touristischen

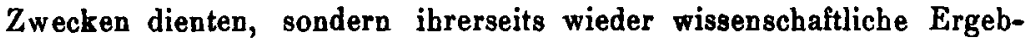
nisse andrer Art zeitigten.

Man hätte glauben Bollen, daß eine unter so glücklichen Auspizien begonnene wissenschaftliche Laufbahn nun in gerader Richtung aufwärts führen und auch alle die äußeren Erfolge, die auf diesem Wege liegen, mühelos erreichen sollte, und man möchte annehmen, daß es so gekommen wäre, wenn Weyl unbeirrt in der eingeschlagenen Richtung verbarrt hätte. Aber sei es, daß sich seine Stellung nicht auf die Dauer den ursprünglichen Hoffnungen entsprechend gestaltete, sei es, daß auch andre Einflüsse mitwirkten, um ihm das Leben in dem fränkischen Universitätsstädtchen zu verleiden, nach kaum dreijährigem Aufenthalt in Erlangen legte Weyl seine dortige Stellung nieder und ging nach Berlin zurück. Hier errichtete er zunächst ein eigenes Laboratorium, in dem er selbst wissenschaftlich arbeitete, aber auch belreundeten Gelehrten, die sein reges wissenschaftliches Interesse anzog, für eigene Studien Gelegenbeit gab. Dort entstanden Untersuchungen süber die Bestimmung des Kuhcaseins durch Fällung mit Schwefelsäure ${ }^{1}$ ), »über den Fettgehalt patbologischer Organe "), über die Nitrate des Tier- und Pflanzenkörpers* ${ }^{3}$ ) nebst einer erleichternden Modilikation für die Bestimmung der Nitrate im Harn «") und wäber die Beziehungen des Cholestearins zu den Terpenen und Campherarten ${ }^{5}$ ). In letzterer Arbeit bestimmte er die Dampidichte dreier, ursprünglich im Jahre 1848-1849 von $\mathrm{Z}$ wenger aus Cholesterin durch Wasserentziehung erbaltener Kohlenwasserstoffe, denen ihr Entdecker die Formel $\mathrm{C}_{26} \mathrm{H}_{43}$ oder $\mathrm{C}_{25} \mathrm{H}_{40}$ gegeben hatte. Weyl fand auffallenderwejse eive Molekulargröße, die

1) Mit I. Frenzel, H. 9, 246 [1885].

3) Mit L. Apt, Virchows Archiv f. patholog. Anatomie u. Physiologie 95, 351 [1884].

3) Ebenda 96, 462 [1884]; mit Citron, ebenda 101, 175 [1885]; mit. W. Gossels, ebenda 105, 187 [1886].

4) Mit A. Meyer, Pflũ gers Archiv f. d. ges. Physiol. 86, 456 [1885̃].

s) Archiv f. Anat. u. Pbysiol., Abt. f. Physiol. 1886, 182. 
nur dem fünften Teil der durch die Formel dargestellten entspricht $\left(\mathrm{C}_{5} \mathrm{H}_{8}\right)$, erbielt aber auch für eine bei $154-158^{\circ}$ siedende Fraktion käuflichen Terpentinöls eine gleiche Molekulargröße $\left(\mathrm{C}_{s} \mathrm{H}_{8}\right)$, für Campher dagegen die normale $\left(\mathrm{C}_{10} \mathrm{H}_{16} \mathrm{O}\right)$. Der von Weyl aus diesen Beobachtungen gezogene Schlu $B$, daß das Cholesterin mit der Reihe der Terpenkörper verwandt sei, hat durch spätere Untersuchungen ${ }^{1}$ ) andrer Chemiker Bestätigung gefunden.

Ganz andren Fragen aber wandte sich Weyls stets reger Geist dann $z u$, und fast von selbst, so möchte man glauben, wurde der zugleich medizinisch und chemisch rorgebildete Forscher auf Probleme hingewiesen, die damals sozusagen in der Luft lagen und auch das Interesse der Behörden sowohl des Staates wie der großen Gemeinwesen mehr und mehr auf sich zogen: die der öffentlichen Gesundheitspflege. Es war die Zeit, da Robert Koch seine ersten Triumphe feierte und der Welt mit Bekanntgabe seiner bakteriologischen Methoden die Wege wies, auf denen man so manche der völkerverderbenden Infektionskrankbeiten durch Frühdiagnose bei ibren Opfern aufspüren, durch das Studium ihrer Erreger in den Bahnen ihrer Verbreitung verfolgen und durch sachgemäBe Vorbeugungsmaßregeln wirksam bekämpfen konnte. Die ersten Untersuchungen Weyls, die sich auf hygienische Fragen beziehen, bewegten sich auf dem Gebiete der giftigen, aber doch dem gewerblichen Verkehr freigegebenen Farbstoffe, wohin besonders sein Feldzug gegen das damals als 2 Safran-Surrogat* gebräuchliche Dinitro-kresol und das Martiusgelb') gehört, sowie eine Studie uber die Wirkung von Chrysarobin und Anthrarobin ") auf den menschlichen Organismus. $\mathrm{DaB}$ er aber Interesse und Freude an rein chemischen Arbeiten nicht verloren hatte, beweisen zwei im Jahre 1888 erschienene Abhandlungen „Zur Kenntnis der Seidek'), die Weyl aus dem Chemischen Institut der Technischen. Hochschule datierte, in das er inzwischen nach Auflösung des eigenen Laboratoriums übergesiedelt war. Von einer alten Beobachtung Mulders aus dem Jahre 1838 ausgehend, nach der Seide in kalter rauchender Salzsäure löslich ist, prufte Weyl, was bei dieser Behandlung aus der Seide wird. Es ließ sich feststellen, $d a B$ ein in festem Zustande isolierbarer Körper, sSericoink, entstanden war, der sich nur wenig ron dem angewandten Fibroin

1) Vergl. z. B. Windaus and Stein, B. 87, 3699 [1904]; Mauthner, M. 28, 1113 [1907].

ग) B. 20,2835 [1887]; 21, 512, 2191 [1888].

ग) Pflü gers Archiv f. d. ges. Physiol. 48, 367 [1888].

) B. 21, 1407, 1529 [1888]. 
der Seide unterschied. Eingreifende Zersetzung aber erleidet das Fibroin durch die Wirkung kochender verdünnter Schwefelsăure und zerfällt dabei in eine Reihe von Aminosäuren, von denen Wesl Tyrosin, Glykokoll und Alanin ( als $\alpha$-Alanin erkannt) in Substanz nachwies. Frübere Bearbeiter dieses Themas (Waltenberger, Städeler, Cramer) hatten durch Hydrolyse mit Schwefelsäure Leucin neben Tyrosin und Glykokoll, mit Barythydrat (Schützenberger und Bourgeois in einer kurzen Notiz) Tyrosin, wein Gemisch gleicher Gewichtsteile Glykokoll und Alanin*, Aminobuttersäure und eine der Acrylsäure-Reihe zugehörige Aminosäure mit vier Kohlenstoffatomen erhalten. Das von Weyl hier zuerst mit Sicherheit als Zersetzungsprodukt eines Proteids nachgewiesene Alanin erkannte E. Fischer ein Jahrzehnt später bei seiner grundlegenden Durchforschung der Eiweißkörper als $d$-Alanin.

Da sich aber, wie schon hervorgehoben, sein Hauptinteresse jetzt mehr und mehr dem Studium der öffentlichen Gesundheitspflege zuwandte, er auch wohl boffte, auf diesem der Wissenschaft neu erschlossenen Gebjete sich eine auch äuBerlich gefestigte Stellung zu schaffen, trat Weyl in das damals neu begründete und unter Kocbs Leitung gestellte Hygienische Institut der Berliner Universität ein, um sich unter diesem, von ihm hochverehrten Forscher mit dessen Arbeitsmethoden vertraut zu machen. Aus den Jahren 1888-1891, die er dieser Tätigkeit widmete, stammen die folgenden Untersuchungen: „Über spon-

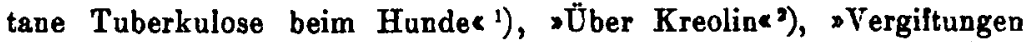
durch Baụmwolle, die mit chromsaurem Blei gefärbt ist « ${ }^{3}$ ), >Über Safranin-Vergiftung ${ }^{4}$ ), ${ }^{2}$ Die Teerfarben mit besonderer Berücksichtigung auf Schädlichkeit und Gesetzgebung ( ${ }^{5}$ ), $Z$ Zur Kenntnis der Anaeroben ${ }^{6}{ }^{6}$ ), - Über Gewinnung von Dauermilch ${ }^{7}$ ), *Zur Kenntnis der Lymphe ${ }^{8}$ ), *Zur Chemie und Toxikologie des Tuberkelbacillusk ${ }^{9}$ ), Zur Theorie der Immunität gegen Milzbrand ${ }^{10}$ ).

Die Titel dieser Arbeiten kennzeichnen schon in gewissem Maße das Arbeitsgebiet, auf dem Weyls geistige Produktion sich nun be-

1) Uhlworms Zentralbl.f. Bakteriologie und Parasitenkunde 6, 689 [1889].

2) Ztschr. f. Hygiene und Infektionskrankheiten 6, 151 [1889].

3) Ztschr. f. Hygiene und Infektionskrankheiten 6, 369 [1889].

4) Ebenda 6, 35 [1889]. 5) Berlin 1889.

6) Mit Kitasato, Ztschr. f. Hygiene 8, 41, 404 [1890]; 9, 97 [1890].

7) Mit R. Pictet, Berl. Klin. Wochenschr. 28, 1009 [1891].

8) Mit M. Schulz, Ztschr. f. Hygiene 10, 523 [1891].

9) Deutsche Medizin. Wochenschr. 17, 256 [1891].

10) Ztschr. f. Hygiene und Infektionskrankheiten 11, 381 [1892]. 
tätigte; neben die darin erwähnten Fragen der Hygiene trat im Laufe dieser und der folgenden Jabre dann noch manche andre, die ihn beschältigte und ibm zu Untersuchungen und Anregungen Anlab bot. Als solche wäre besonders zu nennen die Beseitigung des Hausmülls, wofür er die vor allem in London, aber auch sonst gebräuchliche Verbrennung und damit völlige Unschädlichmachung der Haushaltsabfälle auch für Berlin einzufübren strebte, was sich freilich bei der ganz andren $\mathrm{Zu}$ sammensetzung des Berliner Materials nicht in dieser Weise realisieren ließ. Auch eine Beseitigung der Fäkalien durch Verbrennung regte er an und gab einen dazu geeigneten, in erster Linie für Kasernen, Krankenhäuser usw. bestimmten Apparat an.

Eine seinerzeit ron Werner Siemens im Zusammenhange mit der Konstruktion seines Ozonapparates ausgesprochene, aber nicht zur Durchlührung gebrachte Idee, das Trinkwasser der städtischen Leitungen durch $\mathrm{Ozon}$ von schädlichen Keimen zu befreien, nahm Weyl wieder auf und billete in Gemeinscbalt mit der Firma Siemens ¿ Halske die dazu geeignete Apparatur aus. Die Überführung dieser Methode in die Praxis, die zuerst bei den Interessenten heftigen Widerspruch erfubr, ist seither in einer Reihe von Städten vor sich gegangen.

Eine posthume Genugtung erfuhr eine andre Anregung Weyls. Im Hiablick auf die günstigen Erfahrungen in andren großen Städten hatte er zum ersten Male schon 1891') angeregt, den Winterschnee von den Straßen auch in Berlin, statt ibn viele Kilometer weit hinausfahren und vor der Stadt abladen zu lassen, kurzer Hand in die Wasserläufe resp. die Straßenkanäle zu werfen, und auf diesen Vorschjag kam er dann später ${ }^{2}$ ) wiederholt zurück, ohne daß es ihm aber gelang, die Widerstände der maßgebenden städtischen Persönlichkeiten zu überwinden. Dann aber im letzten Winter, ein halbes Jahr nach Weyls Tode, konnte man in Berliner Zeitungen lesen, daß der Magistrat jetzt angefangen babe, die Schneemassen von den Straßen in die Kanäle werfen statt abfahren zu lassen.

Für alle diese Ideen und so manche andre agitierte er lebbaft durch Wort und Schrift und kam durch diese Tätigkeit, sowie auch durch die viele Jahre von ihm bekleidete Stellung als Sekretär des Berliner »Vereins für öffentliche Gesundheitspflege* mit zablreichen Vertretern der wissenschaftlichen und praktischen Hygiene im In- und Auslande in persőnliche Beziehungen, die ihm manche Mitgliedschaft und Ehrenmitgliedschaft auswärtiger wissenschaftlicher Vereinigungen

J) Th. Weyl, Studien zur Straßenhygiene, Jena 1893.

2) Ges.-Ing. 1898, Nr. 23. 
eintrug. Ebenso wurde er bäufig in bygienischen Fragen durch Prirate und Bebörden um -Gutachten angegangen. Von den Erlebnissen auf einer dieser Reisen pflegte er im Freundeskreise mit besonderem Vergnügen zu erzählen. Er war im Jahre 1896 als Gutachter nach Moskau gegangen, hatte daran eine Reise ins südliche Rußland geschlossen und war so schließlich bis Konstantinopel gelangt. Hier war irgendwie den Behörden von der Ankunft des abendländischen Hygienikers Mitteilung geworden und so nabm man Gelegenheit, ibn die Teile der Stadt sehen zu lassen, wo Cholera und Pest am meisten zu wüten pflegen - wenn sie je ganz aulbören -, um seinen Rat wegen möglicber Abhilfe zu hören. Was er dort sah, hat er in einer kurzen Skizze im »Tag« (vom 27. Juli 1901) veröffentlicht; es ist geeignet, jedem Europäer die Haare zu Berge stehen zu machen. Auch vom Sultan, dem später enttbronten A bdul-Hamid, wurde er damals - es war kurz vor den berüchtigten Armeniermetzeleien - empfangen; er war aber vorber dahin instruiert worden, bei seinen Vorschlägen zur Verbesserung der Zustände die Erwähnung unterirdischer Kanäle sorgfältig zu vermeiden, weil dadurch bei dem argwöhnischen Herrscher nur die Furcht vor Verschwörungen und Überfällen geweckt und die Ausfährung dieser Projekte von vornherein unmöglich gemacht werden würde. Seine Ratschläge wurden durch Verleihung des türkischen Osmanié-Ordens honoriert, ob aber irgend etwas davon zur Ausfübrung kam, ist nicht bekant geworden.

Es ist einleuchtend, daß die so gesammelten zahlreichen Kenntnisse und tatsächlichen Erfahrungen wiederum den Anreiz boten, sich darüber literarisch auszusprechen, und Weyl war wie wenige geneigt und imstande, solcher Anregung Folge zu leisten. Die vielen Übersichten, Zusammenstellungen und Vorschläge tiber alle möglichen Fragen aus der öffentlichen Gesundheitspflege, die in jenen Jahren seiner Feder entflossen, können bier nicht einzeln aufgezäblt werden ${ }^{1}$ ), es sei pur bingewiesen auf einige große Sammelwerke, für die er den Plan aufstellte, die geeignetsten Verfasser für die einzelnen Kapitel anwarb, die Redaktion führte und, als einer seiner fleißigsten Mitarbeiter, wesentliche Bruchteile selbst abfaßte. So entstand das große $\gg$ Handbuch der Hygienex, das in erster Auflage ${ }^{2}$ ) zehn Bände umfaßte und dessen rasch nötig gewordene zweite Auflage er zum großen Teil noch selbst vorbereiten konnte; ferner das $>\mathrm{Handbuch}$ der Arbeiterkrankbeiten $\alpha^{3}$ ), die "Fortschritte der Straßenbygiene ${ }^{4}$ ) und die Assanierung der Städte in Einzeldarstellungen ${ }^{j}$ ).

1) Vergl. die ausführliche Bibliographie von Dipl.-Ing. B ru no Weyl in Jahresber. über Soziale Hygiene, Demographie u. Medicinalstatistik, Bd. 13, [1914].

2) Jena 1896-1901, Suppl.-Bd. I-IV, 1901-1904. 3) Jena 1908.

4) Jena 1901 s) Leipzig 1906-1907. 
Bei letzterer bildet der von ihm selbst herrührende Band über Neapel gewissermaßen den Dank Weyls an diese Stadt für die vielen schönen Stunden, die er ihr verdankte.

1895 habilitierte er sich an der Berliner Technischen Hochschule und las ein stark besuchtes Kolleg $" H y g i e n e$ für Architekten und Ingenieurex, noch mehr aber befriedigten ihn die Vorträge über $\$$ Hygiene für Verwaltungsbeamtex, die er jahrelang als Dozent an der Vereinigung für staatswissenschaftliche Fortbildung hielt. Daneben hatte er einen ständigen Arbeitsplatz im Laboratorium, da die experimentelle Bearheitung chemischer Probleme ibn lebenslänglich fesselte. Da geschah es denn oft, daB er den Vormittag im Laboratorium verbrachte, nachdem er rorher zu Hause schon Korrekturen gelesen oder mit seinen Mitarbeitern korrespondiert hatte, am Nachmittag in einer öffentlichen Bibliotbek historischen Studien über bygienische Fragen oblag, dann einige Stunden als böchst anregender und amüsanter Gesellschafter mit Freunden verbrachte oder wissenschaftlichen Versammlungen beiwohnte und schließlich noch weit über Mitternacht am heimischen Stehpult wieder schriftlich tätig war. Überhaupt kann es kaum einen arbeitsfähigeren und arbeitsfreudigeren Menschen geben, als Theodor Weyl war, und es gehörte sein ganzer Optimismus dazu, um trotz der geringen äußeren Erfolge, die zu erreichen ihm vergönnt war, immer - wenigstens nach außen hin - den guten Humor zu behalten, der ihn seinen Freunden so wert machte. Nur gelegentlich machte sich eine bittere Empfindung in einer sarkastischen Bemerkung Luft.

Im. Jahre 1911 erhielt er den Professortitel; es hieb einige Male, da $\measuredangle$ er für einen akademischen Lehrotubl der Hygiene in Frage gekommen sei, zur Verwirklichung aber ist keine dieser Hoffnungen gediehen.

Von den - meist nur kurzen - Arbeiten auf chemischem Gebiete, die Weyl in dieser letzten Periode seines Lebens veröffentlichte, seien noch genannt eine Notiz über Die Unterscheidung von Ozon,

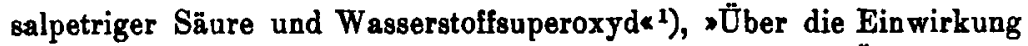
des Ozons auf schwefelhaltige Körper und Schwefel " 2), *Über einen ejnfachen Apparat zur Bestimmung des Schmelzpunktse'), 'Utber das Verbalten von Eiweißkörpern zu Aceton «"), UÜber Reduktionen mit amorphem Phosphor ${ }^{5}$ ); einige weitere Probleme, die ihn zuletzt beschäftigten, sind ungelöst geblieben. Dazu traten von rein literarischen Arbeiten mehrere Bände Betriebsführung städtischer Werke ${ }^{6}$ ) und
1) Mit G. Erlwein, B. 31, 3158 [1899].
2) Ch. Z. 25, 27 [1901].
3) Ch. Z. 34, 488 [1910].
4) B. 48, 508 [1910]; H: 65, 246 [1910].
5) B. 39, $4340[1906] ; 40,970,3608$ [1907].
6) Leipzig 1909-1911. 
-Einzelschriften zur chemischen Technologie «1), an denen er vur als Herausgeber beteiligt war, und eudlich als letztes großes Geschenk an die auf dem Felde der organischen Chemie arbeitenden Chemiker das dreibändige Sammelwerk \#Methoden der organischen Chemie $\times^{3}$ ), in dem er in Gemeinschaft mit einer Anzahl spezialistisch geschulter Mitarbeiter das ganze Gebiet der experimentellen Laboratoriums-Erfahrungen systematlsch in mustergültiger Weise zusammenfaßte.

Ende März 1913 mußte er krank mitten aus einer Besichtigungsreise, die er für ein Gutachten (über den Einfluß des Methylalkohols auf die Gesundheit der damit hantierenden Heimarbeiter) unternommen hatte, heimkehren. Ein schweres Leberleiden, das sich wohl schon lange unerkannt vorbereitet hatte, fesselte ibn an das Krankenzimmer und machte nach einigen sehr schweren Wochen am 6. Juni des gleichen Jahres dem Leben des hochbegabten und bis zum letzten Atemzuge tätigen Mannes ein Ende.

E. Börnstein.

1) Leipzig 1911. 2) Leipzig .1909-1911. 